\title{
On constructive investigation of a class of non-linear boundary value problems for functional differential equations
}

\section{A. RONTÓ and M. RONTÓ}

\section{ABSTRACT.}

We consider a two-point boundary value problem for a non-linear system of functional differential equations, for which a scheme of efficient investigation using certain iteration process is constructed. A new convergence condition is established in the case where a certain additional property of the Lipschitz operator is present, which may lead one to weaker assumptions.

\section{INTRODUCTION}

We consider a two-point boundary value problem for a non-linear system of functional differential equations, for which a scheme of efficient investigation using certain iteration process is constructed. In a sense, this is a continuation of the recent work [9], where the problem is dealt with under different assumptions on the non-linearity.

The aim of this note is to provide an alternative convergence condition in the case where a certain additional property of the Lipschitz operator is present, which may lead one to weaker assumptions.

We recall that, in contrast to numerous approaches using degree theory or fixed point theory (see, e.g., references in [1- $-3,11])$, the method considered allows one to construct approximate solutions and, moreover, rigorously prove the existence of an exact solution by using the results of computation. Mor details on the techniques discussed here can be found in $[4,8,10]$.

\section{NOTATION}

The following notation is used in the sequel:

(1) $C\left([a, b], \mathbb{R}^{n}\right)$ is the Banach space of continuous functions $[a, b] \rightarrow \mathbb{R}^{n}$ with the standard uniform norm.

(2) $L_{1}\left([a, b], \mathbb{R}^{n}\right)$ is the usual Banach space of the vector functions $[a, b] \rightarrow \mathbb{R}^{n}$ with Lebesgue integrable components.

(3) $\mathcal{L}\left(\mathbb{R}^{n}\right)$ is the algebra of all the square matrices of dimension $n$ with real elements.

(4) $r(Q)$ is the maximal in modulus eigenvalue of a matrix $Q \in \mathcal{L}\left(\mathbb{R}^{n}\right)$.

(5) $\mathbf{1}_{n}$ is the unit matrix of dimension $n$.

(6) $\mathbf{0}_{n}$ is the zero square matrix of dimension $n$.

(7) For any $x_{i}, i=1,2, \ldots, n$, we use the notation $\operatorname{col}\left(x_{1}, x_{2}, \ldots, x_{n}\right)$ and $x=\left(x_{i}\right)_{i=1}^{n}$ for the column vector constituted by $x_{1}, x_{2}, \ldots, x_{n}$.

Received: 30.10.2011; In revised form: 02.09.2012; Accepted: 15.10.2012

2010 Mathematics Subject Classification. 34B15.

Key words and phrases. Functional differential equation, two-point boundary conditions, successive approximations, convergence, existence.

Corresponding author: A. Rontó; ronto@math.cas.cz 
(8) $\partial \Omega$ is the boundary of a set $\Omega \subset \mathbb{R}^{n}$.

(9) For any vectors $v_{i}, i=1,2, \ldots, n$, we denote by $\left[v_{1}, v_{2}, \ldots, v_{n}\right]$ the $n \times n$ matrix with the columns $v_{1}, v_{2}, \ldots, v_{n}$.

(10) For $u$ and $v$ from $\mathbb{R}^{n}$, we put $\langle u, v\rangle:=\left\{x \in \mathbb{R}^{n} \mid u \leq x \leq v\right\}$.

(11) For any $x \in \mathbb{R},[x]_{-}:=-\min \{x, 0\}$ and $[x]_{+}:=\max \{x, 0\}$.

(12) $\operatorname{deg} F$ is the Brower degree of a vector field $F$.

(13) By $e_{i}, i=1,2, \ldots, n$, we denote the $n$-dimensional unit vectors

$$
e_{i}:=\operatorname{col}(\underbrace{0,0, \ldots, 0}_{i-1}, 1,0, \ldots, 0) .
$$

(14) Given a linear operator $l=\left(l_{i}\right)_{i=1}^{n}: C\left([a, b], \mathbb{R}^{n}\right) \rightarrow L_{1}\left([a, b], \mathbb{R}^{n}\right)$, we define its components $l_{i k}: C\left([a, b], \mathbb{R}^{n}\right) \rightarrow L_{1}\left([a, b], \mathbb{R}^{n}\right), i, k=1,2, \ldots, n$, by putting

$$
\left(l_{i k} u\right)(t):=l_{i}\left(u e_{k}\right), \quad t \in[a, b],
$$

for all $i, j=1,2, \ldots, n$ and $u \in C([a, b], \mathbb{R})$.

(15) $\left\langle z_{0}, z_{1}\right\rangle$ and $\mathcal{B}\left(y_{0}, y_{1}\right)$ : see (4.8) and (4.13).

\section{PRoblem SETting}

We consider the system of functional differential systems

$$
x^{\prime}(t)=(f x)(t), \quad t \in[a, b],
$$

determined by a non-linear operator $f: C\left([a, b], \mathbb{R}^{n}\right) \rightarrow L_{1}\left([a, b], \mathbb{R}^{n}\right)$. Equation $[3.3)$ is studied under the two-point linear boundary conditions of a non-separated type

$$
A x(a)+B x(b)=d,
$$

where $B$ is a non-singular matrix.

Without loss of generality, one may restrict oneself to the boundary condition of the particular form

$$
A x(a)+x(b)=0 .
$$

For the latter purpose, it is sufficient to introduce a new variable according to the formula

$$
y(t)=B x(t)-\frac{t-a}{b-a} d, \quad t \in[a, b],
$$

and make use of the fact that $B$ is non-singular. Then, clearly, for any $x$ satisfying (3.4), the corresponding function 3.6 has the property

$$
A B^{-1} y(a)+y(b)=0
$$

and, conversely, for any $y$ with property (3.7), the function

$$
x(t)=B^{-1} y(t)+\frac{t-a}{b-a} B^{-1} d, \quad t \in[a, b],
$$

satisfies condition (3.4).

In what follows, skipping the explicit change of variable (3.6), we replace the boundary condition (3.4) by (3.5) and deal with problem (3.3), 3.5) directly. 


\section{SUCCESSIVE APPROXIMATIONS AND MAIN ASSUMPTIONS}

We look for a solution of problem 3.3, 3.5) among functions having initial value in the cone segment $\left\langle z_{0}, z_{1}\right\rangle$ of the form

$$
\left\langle z_{0}, z_{1}\right\rangle:=\left\{z \in \mathbb{R}^{n} \mid z_{0} \leq z \leq z_{1}\right\},
$$

where $z_{0}$ and $z_{1}$ are fixed vectors. Here and below the inequalities for vectors and matrices are understood in the componentwise sense.

Definition 4.1. An operator $l: C\left([a, b], \mathbb{R}^{n}\right) \rightarrow L_{1}\left([a, b], \mathbb{R}^{n}\right)$ is said to be positive if $(l u)(t) \geq 0$ for a.e. $t \in[a, b]$ whenever $u(t) \geq 0$ for all $t \in[a, b]$.

Definition 4.2. An operator $f: C\left([a, b], \mathbb{R}^{n}\right) \rightarrow L_{1}\left([a, b], \mathbb{R}^{n}\right)$ is said to satisfy the Lipschitz condition on a set $\mathcal{B} \subset C\left([a, b], \mathbb{R}^{n}\right)$ if there exists a positive linear operator $l: C\left([a, b], \mathbb{R}^{n}\right) \rightarrow L_{1}\left([a, b], \mathbb{R}^{n}\right)$ such that

$$
|(f u)(t)-(f v)(t)| \leq(l|u-v|)(t), \quad t \in[a, b],
$$

for all $u$ and $v$ from $\mathcal{B}$.

Given any vectors $y_{0}$ and $y_{1}$ from $\mathbb{R}^{n}$, we define the set $\mathcal{B}\left(y_{0}, y_{1}\right)$ by putting

$$
\mathcal{B}\left(y_{0}, y_{1}\right):=\left\{x \in C\left([a, b], \mathbb{R}^{n}\right): y_{0} \leq x(t) \leq y_{1} \text { for all } t \in[a, b]\right\} .
$$

Our study of solutions of the boundary value problem $(3.3),(3.5)$ is based upon the use of the function sequence determined by the recurrence relation

$$
x_{m+1}(\cdot, z):=\operatorname{Pf} x_{m}(\cdot, z)+\varphi_{z}, \quad m=0,1,2, \ldots, z \in\left\langle z_{0}, z_{1}\right\rangle,
$$

with $x_{0}(\cdot, z):=\varphi_{z}$, where

$$
\varphi_{z}(t):=z-\frac{t-a}{b-a}\left(A+\mathbf{1}_{n}\right) z, \quad t \in[a, b] .
$$

and

$$
(P y)(t):=\int_{a}^{t} y(s) d s-\frac{t-a}{b-a} \int_{a}^{b} y(s) d s, \quad t \in[a, b] .
$$

The vector $z$ in (4.11) is considered as an unknown parameter. It can be easily verified that, for every $m=0,1,2, \ldots$ function (4.11) satisfies the boundary condition (3.5) for an arbitrary $z \in \mathbb{R}^{n}$.

Let us introduce into consideration the $n \times n$ matrices

$$
\bar{A}_{-}=\left(\bar{a}_{-; i, j}\right)_{i, j=1}^{n} \text { and } \overline{\bar{A}}_{-}=\left(\overline{\bar{a}}_{-; i, j}\right)_{i, j=1}^{n}
$$

with the elements defined by the equalities

$$
\bar{a}_{-; i, j}:= \begin{cases}0 & \text { if } i \neq j \\ \min \left\{1,\left[a_{i i}\right]_{-}\right\} & \text {if } i=j\end{cases}
$$

and

$$
\overline{\bar{a}}_{-; i, j}:= \begin{cases}{\left[a_{i j}\right]_{-}} & \text {if } i \neq j \\ \max \left\{1,\left[a_{i i}\right]_{-}\right\} & \text {if } i=j\end{cases}
$$

Finally, we put

$$
\omega(z):=\operatorname{ess}_{t \in[a, b]} \sup \left(f \varphi_{z}\right)(t)-\operatorname{essinf}_{t \in[a, b]}\left(f \varphi_{z}\right)(t)
$$

for all $z \in\left\langle z_{0}, z_{1}\right\rangle$, where $\varphi_{z}$ is the function defined by 4.12. 


\section{CONVERGENCE OF SUCCESSive APPROXIMATIONS}

Throughout the paper, we consider the case where the positive linear operator $l: C\left([a, b], \mathbb{R}^{n}\right) \rightarrow L_{1}\left([a, b], \mathbb{R}^{n}\right)$ appearing in the Lipschitz condition (4.9) possesses the property

$$
\underset{t \in[a, b]}{\operatorname{ess} \sup } \frac{\left(l_{i j} \sigma\right)(t)}{\sigma(t)}<+\infty
$$

for all $i, j=1,2, \ldots, n$, where $\sigma$ is the function given by the formula

$$
\sigma(t):=(t-a)(b-t), \quad t \in[a, b] .
$$

Here and below, $l_{i j}: C([a, b], \mathbb{R}) \rightarrow L_{1}([a, b], \mathbb{R}), i, j=1,2, \ldots, n$, are the components of $l$ defined according to (2.2).

It is clear that, under assumption (5.17), the values

$$
v_{i j}:=\operatorname{ess}_{t \in[a, b]} \frac{1}{\sigma(t)}\left(l_{i j} \sigma\right)(t)
$$

are finite for any $i, j=1,2, \ldots, n$. We denote the square matrix $\left(v_{i j}\right)_{i, j=1}^{n}$ by $V$. Since $\sigma$ is a non-negative function and the operator $l$ is positive, it is clear that all the elements of $V$ are non-negative.

Theorem 5.1. Assume that $f$ satisfies the Lipschitz condition (4.9) on the set

$$
\mathcal{B}\left(-\varrho_{*}+\bar{A}_{-} z_{0}-A_{+} z_{1}, \overline{\bar{A}}_{-} z_{1}+\varrho_{*}\right),
$$

where

$$
\varrho_{*}:=\frac{3}{4}\left(\frac{3}{b-a} \mathbf{1}_{n}-V\right)^{-1} \sup _{\xi \in\left\langle z_{0}, z_{1}\right\rangle} \omega(\xi)
$$

and $l: C\left([a, b], \mathbb{R}^{n}\right) \rightarrow L_{1}\left([a, b], \mathbb{R}^{n}\right)$ is a certain positive linear operator with property (5.17). Furthermore, assume that the corresponding matrix $V=\left(v_{i j}\right)_{i, j=1}^{n}$ with elements (5.19) satisfies the condition

$$
r(V)<\frac{3}{b-a} .
$$

Then:

(1) For any fixed $z \in\left\langle z_{0}, z_{1}\right\rangle$, the sequence of functions (4.11) converges uniformly on $[a, b]$ to a function

$$
x_{\infty}(\cdot, z):=\lim _{m \rightarrow \infty} x_{m}(\cdot, z)
$$

possessing the properties

$$
\begin{gathered}
x_{\infty}(a, z)=z \\
A x_{\infty}(a, z)+x_{\infty}(b, z)=0 .
\end{gathered}
$$

(2) The formula

$$
\Delta(z):=\left(A+\mathbf{1}_{n}\right) z+\int_{a}^{b}\left(f x_{\infty}(\cdot, z)\right)(s) d s, \quad z \in\left\langle z_{0}, z_{1}\right\rangle,
$$

introduces a well defined single-valued function $\Delta:\left\langle z_{0}, z_{1}\right\rangle \rightarrow \mathbb{R}^{n}$. 
(3) The limit function (5.22) for all fixed $z \in\left\langle z_{0}, z_{1}\right\rangle$ is a solution of the Cauchy problem

$$
\begin{aligned}
& x^{\prime}(t)=(f x)(t)-\Delta(z), \quad t \in[a, b], \\
& x(a)=z,
\end{aligned}
$$

where the vector function $\Delta:\left\langle z_{0}, z_{1}\right\rangle \rightarrow \mathbb{R}^{n}$ is given by (5.25).

(4) For all fixed $z \in\left\langle z_{0}, z_{1}\right\rangle$ and $m \geq 1$,

$$
\max _{t \in[a, b]}\left|x_{\infty}(t, z)-x_{m}(t, z)\right| \leq \sigma(t) \frac{(b-a)^{m-1}}{3^{m}} V^{m} \tilde{V} \omega(z),
$$

where

$$
\tilde{V}:=\left(\mathbf{1}_{n}-\frac{1}{3}(b-a) V\right)^{-1} .
$$

Note that assumption (5.17) is satisfied in many cases. For example, if the components of the Lipschitz operator $l$ in (4.9) have the form

$$
\left(l_{i k} u\right)(t):=p_{i k}(t) u\left(\tau_{i k}(t)\right), \quad t \in[a, b], i, k=1,2, \ldots, n,
$$

with $p_{i k}:[a, b] \rightarrow \mathbb{R}$ integrable and $\tau_{i k}:[a, b] \rightarrow[a, b]$ measurable, it follows immediately from the relation

$$
\frac{\left(l_{i j} \sigma\right)(t)}{\sigma(t)}=p_{i j}(t) \frac{\left(\tau_{i j}(t)-a\right)\left(\tau_{i j}(t)-b\right)}{(t-a)(t-b)}
$$

that (5.17) holds, in particular, if either the function

$$
[a, b] \ni t \longmapsto \frac{p_{i j}(t)}{(t-a)(t-b)}
$$

is essentially bounded or $p_{i j} \in L_{\infty}([a, b], \mathbb{R})$ and $\tau_{i j}(t) \leq t, i, j=1,2, \ldots, n$.

\section{LEMMATA}

Let us put

$$
(H y)(t):=\left(1-\frac{t-a}{b-a}\right) \int_{a}^{t} y(s) d s+\frac{t-a}{b-a} \int_{t}^{b} y(s) d s, \quad t \in[a, b],
$$

for any $y$ from $L_{1}\left([a, b], \mathbb{R}^{n}\right)$.

6.1. Functions $\alpha_{m}, m=0,1, \ldots$. Define the sequence of functions $\left\{\alpha_{m}: m \geq 0\right\}$ by putting $\alpha_{0}:=1$ and

$$
\alpha_{m}:=H \alpha_{m-1}, \quad m=1,2, \ldots
$$

It follows immediately from (4.11) that

$$
\alpha_{1}=\frac{2 \sigma}{b-a},
$$

where $\sigma$ is the function defined by equality (5.18).

Lemma 6.1. The estimate

$$
\alpha_{2}(t) \leq \frac{b-a}{3} \alpha_{1}(t), \quad t \in[a, b],
$$

holds. 
Proof. Let us put

$$
\mu(t):=\frac{\alpha_{2}(t)}{\alpha_{1}(t)}, \quad t \in[a, b] .
$$

A direct computation according to formula 4.11 shows that

$$
\begin{aligned}
\alpha_{2}(t)= & \frac{1}{(b-a)^{2}}\left(\frac{4}{3} t^{4}-\frac{8(a+b)}{3} t^{3}+\left(6 b a+b^{2}+a^{2}\right) t^{2}\right. \\
& \left.+\frac{a+b}{3}\left(a^{2}+b^{2}-10 a b\right) t+\frac{1}{3}\left(6 b^{2} a^{2}-b a^{3}-a b^{3}\right)\right), \quad t \in[a, b],
\end{aligned}
$$

whence, after rearranging and taking 6.33) into account, we bring 6.35) to the form

$$
\mu(t)=\frac{1}{b-a}\left(-\frac{2 t^{2}}{3}+\frac{2(a+b) t}{3}+\frac{1}{6}\left(b^{2}+a^{2}-6 b a\right)\right) \quad t \in[a, b] .
$$

It is clear from 6.36 that $\mu^{\prime}(t)=0$ if and only if $t=(a+b) / 2$. Since

$$
\mu((a+b) / 2)=\frac{b-a}{3}
$$

and $\mu(a)=\mu(b)=(b-a) / 6$, we see that $\max _{t \in[a, b]} \mu(t)=(b-a) / 3$, whence the required estimate 6.34) follows.

Lemma 6.2. For any non-negative vector $\lambda \in \mathbb{R}^{n}$,

$$
(H \sigma \lambda)(t) \leq \frac{b-a}{3} \sigma(t) \lambda, \quad t \in[a, b]
$$

Proof. To obtain (6.37), it will suffice to use 6.33) and 4.11) with $m=2$ and apply Lemma 6.1.

\subsection{Estimates for the values of $P$ and $H$.}

Lemma 6.3 ([6. Lemma 2]). For an arbitrary essentially bounded function $u:[a, b] \rightarrow \mathbb{R}$, the estimate

$$
|(P u)(t)| \leq \frac{\sigma(t)}{b-a}(\underset{s \in[a, b]}{\operatorname{ess} \sup } u(s)-\underset{s \in[a, b]}{\operatorname{essinf}} u(s))
$$

is true, where $\sigma$ is given by (5.18).

Lemma 6.4 ([9]). The estimate

$$
|(P u)(t)| \leq(H|u|)(t), \quad t \in[a, b],
$$

holds for any $u$ from $C\left([a, b], \mathbb{R}^{n}\right)$.

Lemma 6.5. Assume that condition (5.17) holds. Then, for any non-negative vector $\lambda \in \mathbb{R}^{n}$, the componentwise estimate

$$
l(\sigma \lambda)(t) \leq \sigma(t) V \lambda, \quad t \in[a, b],
$$

holds, where $V=\left(v_{i j}\right)_{i, j=1}^{n}$ is the matrix with the elements determined by relations (5.19).

Proof. Due to 5.19), it follows from 5.17) that

$$
v_{i j} \sigma(t) \geq\left(l_{i j} \sigma\right)(t)
$$


for all $i, j=1,2, \ldots, n$. Therefore,

$$
\sum_{j=1}^{n} \lambda_{j}\left(v_{i j} \sigma(t)-\left(l_{i j} \sigma\right)(t)\right) \geq 0, \quad t \in[a, b],
$$

for any $i=1,2, \ldots, n$, and non-negative $\lambda_{j}, j=1,2, \ldots, n$. Estimate (6.40) is a direct consequence of 6.41).

6.3. Estimates of $\varphi_{z}$. For any $z \in\left\langle z_{0}, z_{1}\right\rangle$ and any vector $\varrho \in \mathbb{R}^{n}$ with positive components, we put

$$
\mathcal{A}_{z}(\varrho):=\left\{x \in C\left([a, b], \mathbb{R}^{n}\right):\left|x(t)-\varphi_{z}(t)\right| \leq \varrho \text { for all } t \in[a, b]\right\} .
$$

Lemma $6.6([9])$. $\mathcal{A}_{z}\left(\varrho_{1}\right) \subset \mathcal{A}_{z}\left(\varrho_{2}\right)$ whenever $\varrho_{1} \leq \varrho_{2}$.

For the given matrix $A$ from the boundary condition (3.5), we define its positive and negative parts $A_{+}=\left(a_{+; i, j}\right)_{i, j=1}^{n}$ and $A_{-}=\left(a_{-; i, j}\right)_{i, j=1}^{n}$ by putting

$$
a_{+; i, j}:=\left[a_{i, j}\right]_{+}, \quad a_{-; i, j}:=\left[a_{i, j}\right]_{-}
$$

for all $i$ and $j$ from 1 to $n$. Then, obviously, $A_{+}$and $A_{-}$are non-negative matrices and

$$
A=A_{+}-A_{-} \text {. }
$$

Lemma 6.7 (|9]). For any $z \in\left\langle z_{0}, z_{1}\right\rangle$ and non-negative $\varrho$, the inclusion

$$
\mathcal{A}_{z}(\varrho) \subset \mathcal{B}\left(-\varrho+\bar{A}_{-} z_{0}-A_{+} z_{1}, \overline{\bar{A}}_{-} z_{1}+\varrho\right)
$$

holds.

Recall that $\bar{A}_{-}=\left(\bar{a}_{-; i, j}\right)_{i, j=1}^{n}$ and $\overline{\bar{A}}_{-}=\left(\overline{\bar{a}}_{-; i, j}\right)_{i, j=1}^{n}$ are the matrices with the elements given by formulae (4.14) and (4.15).

\section{ProOf OF THEOREM 5.1}

We shall follow the lines of the proof of [9. Theorem 5.1]. Throughout this section, we fix an arbitrary vector $z$ from the order interval $\left\langle z_{0}, z_{1}\right\rangle$.

7.1. Let us first show that all the members of sequence (4.11) belong to the set

$$
\mathcal{B}\left(-\varrho_{*}+\bar{A}_{-} z_{0}-A_{+} z_{1}, \overline{\bar{A}}_{-} z_{1}+\varrho_{*}\right) .
$$

Indeed, it follows immediately from 6.42 that $\mathcal{A}_{z}(0)=\left\{\varphi_{z}\right\}$ and, therefore, Lemmata 6.6 and 6.7 imply that

$$
\varphi_{z} \in \mathcal{B}\left(-\varrho_{*}+\bar{A}_{-} z_{0}-A_{+} z_{1}, \overline{\bar{A}}_{-} z_{1}+\varrho_{*}\right) .
$$

Since, by definition, $x_{0}(\cdot, z)=\varphi_{z}$, inclusion (7.46), in fact, means that

$$
x_{0}(\cdot, z) \in \mathcal{B}\left(-\varrho_{*}+\bar{A}_{-} z_{0}-A_{+} z_{1}, \overline{\bar{A}}_{-} z_{1}+\varrho_{*}\right) .
$$

Further on, by Lemma 6.3, it follows from 6.31 that

$$
\begin{aligned}
\left|x_{1}(t, z)-\varphi_{z}(t)\right| & =\left|\left(\operatorname{Pf} \varphi_{z}\right)(t)\right| \\
& \leq \frac{\sigma(t)}{b-a} \omega(z), \quad t \in[a, b],
\end{aligned}
$$

where $\sigma$ is the function (5.18) and $\omega:\left\langle z_{0}, z_{1}\right\rangle \rightarrow \mathbb{R}^{n}$ is defined by 4.16. 
Let us put $\varrho_{0}(z):=\frac{1}{4}(b-a) \omega(z)$ and

$$
\varrho_{m}(z):=\frac{b-a}{4}\left(\mathbf{1}_{n}+\frac{1}{3}(b-a) V+\frac{1}{9}(b-a)^{2} V^{2}+\ldots\right.
$$

$$
\left.+\frac{1}{3^{m}}(b-a)^{m} V^{m}\right) \omega(z)
$$

for any $m \geq 1$.

It is clear from 3.3 that

$$
\max _{t \in[a, b]} \sigma(t)=\frac{1}{4}(b-a)^{2}
$$

and, therefore, (7.47) yields

$$
\left|x_{1}(t, z)-\varphi_{z}(t)\right| \leq \frac{b-a}{4} \omega(z)=\varrho_{0}(z), \quad t \in[a, b] .
$$

Hence, according to notation 6.42,

$$
x_{1}(\cdot, z) \in \mathcal{A}_{z}\left(\varrho_{0}(z)\right) .
$$

In view of assumption (5.21, equality (5.20) implies that

$$
\begin{aligned}
\varrho_{*} & =\frac{b-a}{4}\left(\mathbf{1}_{n}-\frac{b-a}{3} V\right)^{-1} \sup _{\xi \in\left\langle z_{0}, z_{1}\right\rangle} \omega(\xi) \\
& \geq \frac{b-a}{4} \sum_{i=0}^{+\infty}\left(\frac{b-a}{3}\right)^{i} V^{i} \omega(z) \\
& =\lim _{m \rightarrow+\infty} \varrho_{m}(z),
\end{aligned}
$$

whence it is clear that

$$
\varrho_{*} \geq \varrho_{m}(z), \quad m=0,1,2, \ldots
$$

Considering (7.53) and applying Lemma 6.7, we obtain the inclusions

$$
\mathcal{A}_{z}\left(\varrho_{m}(z)\right) \subset \mathcal{A}_{z}\left(\varrho_{*}\right) \subset \mathcal{B}\left(-\varrho_{*}+\bar{A}_{-} z_{0}-A_{+} z_{1}, \overline{\bar{A}}_{-} z_{1}+\varrho_{*}\right)
$$

valid for any $m \geq 0$. In view of (7.54), inclusion (7.51) implies that

$$
x_{1}(\cdot, z) \in \mathcal{B}\left(-\varrho_{*}+\bar{A}_{-} z_{0}-A_{+} z_{1}, \overline{\bar{A}}_{-} z_{1}+\varrho_{*}\right) .
$$

Thus, both functions $\varphi_{z}$ and $x_{1}(\cdot, z)$ belong to the set where the Lipschitz condition 4.9 is satisfied. Applying Lemmata 6.4 and 6.5. we get

$$
\begin{aligned}
\left|x_{2}(t, z)-\varphi_{z}(t)\right| & =\mid\left(\operatorname{Pf} x_{1}(\cdot, z)(t) \mid\right. \\
& \leq\left|\left(P f \varphi_{z}\right)(t)\right|+\left|\left(P\left[f x_{1}(\cdot, z)-f \varphi_{z}\right]\right)(t)\right| \\
& \leq \frac{\sigma(t)}{b-a} \omega(z)+\frac{1}{b-a} \operatorname{Hl}(\sigma \omega(z))(t) \\
& \leq \frac{\sigma(t)}{b-a} \omega(z)+\frac{1}{b-a}(H \sigma V \omega(z))(t)
\end{aligned}
$$

for any $t \in[a, b]$.

By Lemma 6.2, the inequality

$$
(H \sigma V \omega(z))(t) \leq \frac{b-a}{3} \sigma(t) V \omega(z), \quad t \in[a, b],
$$


is true. Note that, by (4.16), $\omega(z) \geq 0$ and, hence, $V \omega(z) \geq 0$ as well, so that the estimate of Lemma 6.2 is applicable.

Using the positivity of the operator $H$, applying Lemma 6.5 with $\lambda=\omega(z)$, and taking relations (4.11), 3.3), (7.56), and (7.57) into account, we obtain

$$
\begin{aligned}
\left|x_{2}(t, z)-\varphi_{z}(t)\right| & \leq \frac{\sigma(t)}{b-a} \omega(z)+\frac{1}{b-a} H(\sigma V \omega(z))(t) \\
& \leq \frac{1}{b-a}\left(\sigma(t) \omega(z)+\frac{b-a}{3} \sigma(t) V \omega(z)\right) \\
& =\frac{\sigma(t)}{b-a}\left(\mathbf{1}_{n}+\frac{b-a}{3} V\right) \omega(z), \quad t \in[a, b],
\end{aligned}
$$

whence, by (7.49) and (7.48),

$$
\begin{aligned}
\left|x_{2}(t, z)-\varphi_{z}(t)\right| & \leq \frac{b-a}{4}\left(\mathbf{1}_{n}+\frac{b-a}{3} V\right) \omega(z) \\
& =\varrho_{1}(z)
\end{aligned}
$$

for all $t \in[a, b]$. According to 6.42), estimate (7.59) means that

$$
x_{2}(\cdot, z) \in \mathcal{A}_{z}\left(\varrho_{1}(z)\right) .
$$

In view of inclusions 7.60 and (7.54), the function $x_{2}(\cdot, z)$ belongs to the set $\mathcal{B}\left(-\varrho_{*}+\right.$ $\left.\bar{A}_{-} z_{0}-A_{+} z_{1}, \overline{\bar{A}}_{-} z_{1}+\varrho_{*}\right)$ on which the Lipschitz condition $(4.9)$ is satisfied.

Using (4.9) for the functions $x_{2}(\cdot, z)$ and $\varphi_{z}$, similarly to (7.56) and (7.58), we obtain

$$
\begin{aligned}
\left|x_{3}(t, z)-\varphi_{z}(t)\right| & =\mid\left(\operatorname{Pf} x_{2}(\cdot, z)(t) \mid\right. \\
& \leq\left|\left(\operatorname{Pf} \varphi_{z}\right)(t)\right|+\left|\left(P\left[f x_{2}(\cdot, z)-f \varphi_{z}\right]\right)(t)\right| \\
& \leq \frac{1}{b-a}(\sigma(t) \omega(z)+(H l)(\sigma \omega(z)+(H l)(\sigma \omega(z)))(t)) \\
& =\frac{1}{b-a}(\sigma(t) \omega(z)+(H l(\sigma \omega(z))(t)+(H l H l \sigma \omega(z))(t))
\end{aligned}
$$

and, therefore, by (7.48) and (7.53),

$$
\begin{aligned}
\left|x_{3}(t, z)-\varphi_{z}(t)\right| & \leq \frac{b-a}{4}\left(\mathbf{1}_{n}+\frac{1}{3}(b-a) V+\frac{1}{9}(b-a)^{2} V^{2}\right) \omega(z) \\
& =\varrho_{2}(z) \leq \varrho_{*}, \quad t \in[a, b],
\end{aligned}
$$

whence it follows that $x_{3}(\cdot, z) \in \mathcal{A}_{z}\left(\rho_{2}(z)\right)$ and, consequently, by (7.54),

$$
x_{3}(\cdot, z) \in \mathcal{B}\left(-\varrho_{*}+\bar{A}_{-} z_{0}-A_{+} z_{1}, \overline{\bar{A}}_{-} z_{1}+\varrho_{*}\right) .
$$

Proceeding analogously, we obtain the estimate

$$
\left|x_{m}(t, z)-\varphi_{z}(t)\right| \leq \varrho_{m-1}(z), \quad t \in[a, b],
$$

valid for any $m \geq 1$, whence, in view of (7.54), the inclusion

$$
\left\{x_{m}(\cdot, z): m \geq 0\right\} \subset \mathcal{B}\left(-\varrho_{*}+\bar{A}_{-} z_{0}-A_{+} z_{1}, \overline{\bar{A}}_{-} z_{1}+\varrho_{*}\right)
$$

follows. 
7.2. Let us show that $\left\{x_{m}(\cdot, z): m \geq 0\right\}$ is a Cauchy sequence in the Banach space $C\left([a, b], \mathbb{R}^{n}\right)$.

Indeed, by virtue of definition 4.11 and Lemma 6.4, we have

$$
\begin{aligned}
\left|x_{m+1}(t, z)-x_{m}(t, z)\right| & =\left|\left(P\left[f x_{m}(\cdot, z)-f x_{m-1}(\cdot, z)\right]\right)(t)\right| \\
& \leq H\left|f x_{m}(\cdot, z)-f x_{m-1}(\cdot, z)\right|(t)
\end{aligned}
$$

for all $t \in[a, b]$ and $m \geq 1$. In view of inclusion (7.63), the Lipschitz condition for $f$ holds at all the members of sequence (4.11) and, therefore, estimate (7.64) yields

$$
\begin{aligned}
\left|x_{m+1}(t, z)-x_{m}(t, z)\right| & \leq\left(H l\left|x_{m}(\cdot, z)-x_{m-1}(\cdot, z)\right|\right)(t) \\
& \leq\left((H l)^{m}\left|x_{1}(\cdot, z)-\varphi_{z}\right|\right)(t)
\end{aligned}
$$

for all $t \in[a, b]$ and $m \geq 1$. Due to estimate (7.47), inequality (7.65) yields

$$
\begin{aligned}
\left|x_{m+1}(t, z)-x_{m}(t, z)\right| & \leq \frac{1}{b-a}\left((H l)^{m} \sigma \omega(z)\right)(t) \\
& =\frac{1}{b-a}\left((H l)^{m-1} H \operatorname{lo\omega }(z)\right)(t)
\end{aligned}
$$

for all $m>1$ and $t \in[a, b]$, where $\sigma$ is the function given by (5.18).

By Lemma 6.5.

$$
l(\sigma \omega(z))(t) \leq \sigma(t) V \omega(z), \quad t \in[a, b] .
$$

On the other hand, Lemma 6.2 implies that inequality (7.57) holds,

$$
(H \sigma V \omega(z))(t) \leq \frac{b-a}{3} \sigma(t) V \omega(z), \quad t \in[a, b],
$$

which, together with (7.67), yields

$$
(H l \sigma \omega(z))(t) \leq \frac{b-a}{3} \sigma(t) V \omega(z), \quad t \in[a, b] .
$$

Using (7.68) in (7.66), we get

$$
\begin{aligned}
\left|x_{m+1}(t, z)-x_{m}(t, z)\right| & \leq \frac{1}{b-a}\left((H l)^{m-1} \frac{b-a}{3} \sigma V \omega(z)\right)(t) \\
& =\frac{1}{3}\left((H l)^{m-1} \sigma V \omega(z)\right)(t) .
\end{aligned}
$$

Arguing similarly, we obtain

$$
\begin{aligned}
\left|x_{m+1}(t, z)-x_{m}(t, z)\right| & \leq \frac{1}{b-a} \cdot \frac{b-a}{3}\left((H l)^{m-2} H l \sigma V \omega(z)\right)(t) \\
& \leq \frac{1}{b-a} \cdot \frac{b-a}{3}\left((H l)^{m-2} H \sigma V \cdot V \omega(z)\right)(t) \\
& \leq \frac{1}{b-a}\left(\frac{b-a}{3}\right)^{2}\left((H l)^{m-2} \sigma V^{2} \omega(z)\right)(t)
\end{aligned}
$$

and, finally,

$$
\begin{aligned}
\left|x_{m+1}(t, z)-x_{m}(t, z)\right| & \leq \frac{1}{b-a}\left(\frac{b-a}{3}\right)^{m} \sigma(t) V^{m} \omega(z) \\
& =\frac{1}{3^{m}}(b-a)^{m-1} \sigma(t) V^{m} \omega(z)
\end{aligned}
$$

for all $m \geq 1$ and $t \in[a, b]$. 
By virtue of assumption (5.21), it follows from (7.70) that

$$
\begin{aligned}
\left|x_{m+k}(t, z)-x_{m}(t, z)\right| & \leq \sum_{j=0}^{k-1}\left|x_{m+j+1}(t, z)-x_{m+j}(t, z)\right| \\
& \leq \sigma(t) \sum_{j=0}^{k-1} \frac{(b-a)^{m+j-1}}{3^{m+j}} V^{m+j} \omega(z) \\
& =\sigma(t) \frac{(b-a)^{m-1}}{3^{m}} V^{m} \sum_{j=0}^{k-1} \frac{1}{3^{j}}(b-a)^{j} V^{j} \omega(z) \\
& \leq \sigma(t) \frac{(b-a)^{m-1}}{3^{m}} V^{m} \sum_{j=0}^{+\infty} \frac{1}{3^{j}}(b-a)^{j} V^{j} \omega(z)
\end{aligned}
$$

for any $m \geq 0$ and $k \geq 1$. In view of assumption (5.21), $\lim _{m \rightarrow+\infty} \frac{1}{3^{m}}(b-a)^{m-1} V^{m}=\mathbf{0}_{n}$, and, therefore, estimate (7.71) proves that $\left\{x_{m}(\cdot, z): m \geq 0\right\}$ is a Cauchy sequence in $C\left([a, b], \mathbb{R}^{n}\right)$.

7.3. It is clear from 6.33 that

$$
\varphi_{z}(a)=z, \quad \varphi_{z}(b)=-A z .
$$

Therefore, by 4.11 and 4.13,

$$
\begin{aligned}
& x_{m}(a, z)=\left(P x_{m-1}(\cdot, z)\right)(a)=z, \\
& x_{m}(b, z)=\left(P x_{m-1}(\cdot, z)\right)(b)+\varphi_{z}(b)=-A z,
\end{aligned}
$$

whence it follows that the initial condition

$$
x_{m}(a, z)=z
$$

and the two-point boundary condition

$$
A x_{m}(a, z)+x_{m}(b, z)=0
$$

are satisfied for any $m \geq 1$.

Passing to the limit as $m \rightarrow \infty$ in (7.72, (7.73), we arrive at the required equalities (5.23), (5.24).

Passing to the limit as $m \rightarrow \infty$ in equality 4.11 , we show that the function $x_{\infty}(\cdot, z)$ given by 5.22) is the unique solution the integro-functional equation

$$
x(t)=\varphi_{z}(t)+(P f x)(t), \quad t \in[a, b] .
$$

In particular, the function $\Delta:\left\langle z_{0}, z_{1}\right\rangle \rightarrow \mathbb{R}^{n}$ is well defined by formula (5.25).

Differentiating both sides of (7.74) and recalling 6.31) and 6.33, we find that, for an arbitrary $z \in\left\langle z_{0}, z_{1}\right\rangle$, the function $x=x_{\infty}(\cdot, z)$ is the unique solution of the Cauchy problem 5.26, 5.27.

Finally, passing to the limit as $k \rightarrow \infty$ in (7.71), we obtain the inequality

$$
\left|x_{\infty}(t, z)-x_{m}(t, z)\right| \leq \sigma(t) \frac{(b-a)^{m-1}}{3^{m}} V^{m} \sum_{j=0}^{+\infty} \frac{1}{3^{j}}(b-a)^{j} V^{j} \omega(z),
$$

which coincides with the required estimate (5.28). This completes the proof. 


\section{A REMARK ON CONDITION 5.21}

In [9], the successive approximations (4.11) are considered on the assumption that $f$ in (3.3) satisfies the Lipschitz condition 4.9 ) on the set $\mathcal{B}\left(-\varrho^{*}+\bar{A}_{-} z_{0}-A_{+} z_{1}, \overline{\bar{A}}_{-} z_{1}+\varrho^{*}\right)$ with

$$
\varrho^{*}:=\frac{b-a}{4}\left(1-Q_{l}\right)^{-1} \sup _{z \in\left\langle z_{0}, z_{1}\right\rangle} \omega(z),
$$

where $Q_{l}$ is the constant matrix of the form

$$
Q_{l}:=\max _{t \in[a, b]}\left(\left(1-\frac{t-a}{b-a}\right) \int_{a}^{t} K_{l}(s) d s+\frac{t-a}{b-a} \int_{t}^{b} K_{l}(s) d s\right)
$$

and the matrix-valued function $K_{l}:[a, b] \rightarrow \mathcal{L}(\mathbb{R})$ is associated with a positive linear operator $l: C \rightarrow L_{1}$ according to the formula

$$
K_{l}:=\left[l e_{1}, l e_{2}, \ldots, l e_{n}\right],
$$

with $e_{i}, i=1,2, \ldots, n$, are the unit vectors given by (5.17).

We note that the maximum in 8.76 is taken elementwise, and it is, in general, not attained at a point from $[a, b]$ unless $n=1$.

The convergence of the successive approximations 4.11 has been proven in $[9$. Theorem 5.1] on the condition

$$
r\left(Q_{l}\right)<1 .
$$

In contrast to $(8.78)$, the condition (5.21) used in Theorem 5.1 depends solely on the value of the Lipschitz operator $l$ at the function $\sigma$ and, as is seen from examples, may be weaker in the cases where $l \sigma$ is small in some sense.

\section{ANALYSIS OF SOLVABILITY}

The following general statement on the solvability of the boundary value problem (3.3), (3.5) holds.

Theorem 9.2. Let the conditions of Theorem 5.1 be satisfied. Then the limit function $x_{\infty}(\cdot, z)$ of the recurrence sequence (4.11) is a solution of the boundary value problem (3.3), 3.5) if, and only if the value of the vector parameter $z \in\left\langle z_{0}, z_{1}\right\rangle$ satisfies the system of equations

$$
\Delta(z)=0,
$$

where $\Delta:\left\langle z_{0}, z_{1}\right\rangle \rightarrow \mathbb{R}^{n}$ is given by (5.25).

The proof of Theorem 9.2 is similar to that of [9. Theorem 6.2] and is omitted.

Equations of type 9.79 are usually referred to as "determining equations" because they determine the actual values of the parameters $z \in\left\langle z_{0}, z_{1}\right\rangle$ involved in the iteration process (4.11). Likewise, $\Delta:\left\langle z_{0}, z_{1}\right\rangle \rightarrow \mathbb{R}^{n}$ given by (5.25) is often called a "determining function" for problem (3.3), (3.5).

A constructive investigation of problem (3.3, 3.5 with the help of Theorem 9.2 is carried out by passing from the exact determining equation (9.79) to some its approximations. In practice, it is natural to fix some $m \geq 1$, introduce the $m$ th "approximate determining function" $\Delta_{m}:\left\langle z_{0}, z_{1}\right\rangle \rightarrow \mathbb{R}^{n}$ by setting

$$
\Delta_{m}(z):=\left(A+\mathbf{1}_{n}\right) z+\int_{a}^{b}\left(f x_{m}(\cdot, z)\right)(s) d s, \quad z \in\left\langle z_{0}, z_{1}\right\rangle,
$$


and, instead of the inconvenient equation (9.79), consider the $m$ th "approximate determining equation" of the form

$$
\Delta_{m}(z)=0 .
$$

It should be noted that, in contrast to 9.79, the new equation 9.88) is constructed directly based on the function $x_{m}(\cdot, z)$ and does not involve any unknown terms. It turns out that, under suitable assumptions, the function

$$
X_{m}(t):=x_{m}(t, \tilde{z}), \quad t \in[a, b],
$$

where $\tilde{z}$ iz a root of equation 9.81, can be regarded as an $m$ th approximation to a solution of problem 3.3, 3.5.

Definition 9.3. Let $S \subset \mathbb{R}^{n}$ be an arbitrary non-empty set. For any pair of functions $g_{j}=\operatorname{col}\left(g_{j, 1}, \ldots, g_{j, n}\right), j=1,2$, we write

$$
g_{1} \triangleright_{S} g_{2}
$$

if and only if there exists a function $\nu: S \rightarrow\{1,2, \ldots, n\}$ such that the strict inequality

$$
g_{1, \nu(x)}(x)>g_{2, \nu(x)}(x)
$$

holds for all $x \in S$.

The following statement gives conditions sufficient for the solvability of the boundary value problem (3.3), 6.40 based on properties of a certain fixed member of the recurrence sequence 4.11.

Theorem 9.3. Let us suppose that, in addition to assumptions of Theorem 5.1 there exist a closed domain $\Omega \subset\left\langle z_{0}, z_{1}\right\rangle$ and an integer $m \geq 1$ such that, on the boundary of $\Omega$, the approximate determining function $\Delta_{m}$ given by formula 9.80 satisfies the condition

$$
\left|\Delta_{m}\right| \triangleright_{\partial \Omega} \frac{1}{2} \frac{(b-a)^{m+2}}{3^{m+1}} V^{m+1} \tilde{V} \omega,
$$

where $\omega:\left\langle z_{0}, z_{1}\right\rangle \rightarrow \mathbb{R}^{n}$ is the function given by (4.16) and $\tilde{V}$ is the matrix (5.29).

Let, moreover,

$$
\operatorname{deg}\left(\Delta_{m}, \Omega, 0\right) \neq 0 .
$$

Then there exists a certain $z^{*} \in \Omega$ such that the function $x_{\infty}\left(\cdot, z^{*}\right)$ is a solution of the boundary value problem (3.3, 3.5.

The proof of Theorem 9.3 is analogous to that of [9. Theorem 7.1] and is based on the application of the following

Lemma 9.8. Under the assumptions of Theorem 5.1.

$$
\left|\Delta(z)-\Delta_{m}(z)\right| \leq \frac{1}{2} \frac{(b-a)^{m+2}}{3^{m+1}} V^{m+1} \tilde{V} \omega(z)
$$

for arbitrary $z \in\left\langle z_{0}, z_{1}\right\rangle$ and $m \geq 1$.

Proof. Let $z \in\left\langle z_{0}, z_{1}\right\rangle$ and $m \geq 1$ be arbitrary. By virtue of 5.25 and 9.81, we have

$$
\begin{aligned}
\left|\Delta(z)-\Delta_{m}(z)\right| & =\left|\int_{a}^{b}\left[f x_{\infty}(\cdot, z)(t)-f x_{m}(\cdot, z)(t)\right] d t\right| \\
& \leq \int_{a}^{b}\left|f x_{\infty}(\cdot, z)(t)-f x_{m}(\cdot, z)(t)\right| d t
\end{aligned}
$$


Since condition (5.21) is assumed, it follows that estimate (7.62) is satisfied for any $m \geq 1$. Passing to the limit as $m \rightarrow \infty$ in (7.62) and taking (5.20), (7.52) into account, we obtain

$$
\left|x_{\infty}(t, z)-\varphi_{z}(t)\right| \leq \lim _{k \rightarrow+\infty} \varrho_{k}(z) \leq \varrho_{*}, \quad t \in[a, b],
$$

whence, according to (7.54), we get

$$
x_{\infty}(\cdot, z) \in \mathcal{B}\left(-\varrho_{*}+\bar{A}_{-} z_{0}-A_{+} z_{1}, \overline{\bar{A}}_{-} z_{1}+\varrho_{*}\right) .
$$

It follows from (7.63) and 9.89) that the Lipschitz condition (4.9) imposed on $f$ can be applied for the pair of functions $x_{\infty}(\cdot, z)$ and $x_{m}(\cdot, z)$. By doing so in 9.87), taking estimate 5.28 into account, and applying Lemma 6.5 with $\lambda=V^{m} \tilde{V} \omega(z)$ and $\tilde{V}$ given by (5.29), we obtain

$$
\begin{aligned}
\left|\Delta(z)-\Delta_{m}(z)\right| & \leq \int_{a}^{b} l\left|x_{\infty}(\cdot, z)(t)-x_{m}(\cdot, z)(t)\right| d t \\
& \leq \frac{(b-a)^{m-1}}{3^{m}} \int_{a}^{b}\left(l \sigma V^{m} \tilde{V} \omega(z)\right)(t) d t \\
& \leq \frac{(b-a)^{m-1}}{3^{m}} \int_{a}^{b} \sigma(t) d t V V^{m} \tilde{V} \omega(z) .
\end{aligned}
$$

Since, by 5.18,

$$
\int_{a}^{b} \sigma(t) d t=\frac{1}{6}(b-a)^{3}
$$

relation 9.90 implies the estimate

$$
\left|\Delta(z)-\Delta_{m}(z)\right| \leq \frac{1}{6} \frac{(b-a)^{m+2}}{3^{m}} V^{m+1} \tilde{V} \omega(z),
$$

which coincides with 9.86.

\section{AN EXAMPLE OF APPLICATION}

Consider the functional differential system

$$
\begin{aligned}
& x_{1}^{\prime}(t)=-3 t^{2}\left(x_{2}\left(t^{2}\right)\right)^{2}, \\
& x_{2}^{\prime}(t)=(1-t)^{5}\left(3 x_{1}\left(\frac{t}{3}\right)+\frac{t^{3}}{9}\right), \quad t \in[0,1],
\end{aligned}
$$

with the two-point boundary conditions

$$
\begin{aligned}
x_{2}(0)+x_{1}(1) & =0, \\
x_{1}(0)-x_{2}(0)+x_{2}(1) & =0 .
\end{aligned}
$$

Clearly, system 10.91, 10.92 is a particular case of 3.3 with $a=0, b=1, n=2$,

$$
(f x)(t):=\left(\begin{array}{c}
-3 t^{2}\left(x_{2}\left(t^{2}\right)\right)^{2} \\
(1-t)^{5}\left(3 x_{1}\left(\frac{t}{3}\right)+\frac{1}{9} t^{3}\right)
\end{array}\right), \quad t \in[0,1],
$$

for all $x=\left(x_{i}\right)_{i=1}^{2} \in C\left([a, b], \mathbb{R}^{2}\right)$, whereas the boundary conditions 10.93, (10.94) take form (3.5) if one puts

$$
A:=\left(\begin{array}{cc}
0 & 1 \\
1 & -1
\end{array}\right)
$$


One can verify that the conditions of Theorem 5.1 hold, and it thus makes sense to apply the approach described above. We omit the details and focus on the results of computation. Note only that the coefficients in (10.91), (10.92) are continuous functions and the argument deviations are of delay type and, thus, by virtue of relation (5.30), assumption (5.17) is satisfied.

By (10.96), the starting approximation 4.12) in this case has the form

$$
\varphi_{z}(t)=\left(\begin{array}{c}
z_{1}-t z_{1}-t z_{2} \\
z_{2}-t z_{1}
\end{array}\right), \quad t \in[0,1]
$$

Carrying out the first step of iteration according to formula 4.11 , we obtain the equalities

$$
\begin{aligned}
x_{11}\left(t, z_{1}, z_{2}\right)= & z_{1}-t z_{1}-t z_{2}-\frac{3}{7} z_{1}^{2} t^{7}+\frac{6}{5} z_{2} z_{1} t^{5}-z_{2}{ }^{2} t^{3} \\
& +t\left(\frac{3}{7} z_{1}^{2}-\frac{6}{5} z_{2} z_{1}+z_{2}^{2}\right)
\end{aligned}
$$

and

$$
\begin{aligned}
x_{12}\left(t, z_{1}, z_{2}\right)= & z_{2}+\frac{32}{21} t z_{1}-\frac{1}{81} t^{9}+\frac{5}{72} t^{8}-\frac{10}{63} t^{7}+\frac{1}{7} t^{7} z_{1}+\frac{1}{7} t^{7} z_{2} \\
& -\frac{4}{3} t^{6} z_{1}+\frac{5}{27} t^{6}-\frac{5}{6} t^{6} z_{2}+5 t^{5} z_{1}-\frac{1}{9} t^{5}+2 t^{5} z_{2}-10 t^{4} z_{1} \\
& +\frac{1}{36} t^{4}-\frac{5}{2} t^{4} z_{2}+\frac{35}{3} t^{3} z_{1}+\frac{5}{3} t^{3} z_{2}-8 t^{2} z_{1}-\frac{1}{2} t^{2} z_{2} \\
& -\frac{1}{4536} t+\frac{1}{42} t z_{2}
\end{aligned}
$$

for all $t \in[0,1]$. Constructing the vector function $\Delta_{1}: \mathbb{R}^{2} \rightarrow \mathbb{R}^{2}$ according to 9.80 with $m=1$, we find that the first approximate determining system (9.81) has the form

$$
\begin{gathered}
\frac{35822288758573397}{35821106339668650} z_{1}+\frac{35819006959189709}{35821106339668650} z_{2} \\
+\frac{581323225130404}{663353821104975} z_{2} z_{1}-\frac{5514914693}{3180914242962576120} \\
-\frac{11912489872861}{12060978565545} z_{2}^{2}-\frac{41035461286199}{132670764220995} z_{1}^{2}=0 \\
\frac{31}{21} z_{1}-\frac{1}{42} z_{2}+\frac{1}{4536}-\frac{1069}{37422} z_{2} z_{1}+\frac{107}{4536} z_{2}{ }^{2}+\frac{1250957}{122594472} z_{1}^{2}=0
\end{gathered}
$$

The computation here has been performed using Maple $e^{\mathrm{TM}}$.

Solving system (10.99), (10.100) numerically in a neighbourghood of point $(0,1)$, we obtain

$$
z_{1} \approx-0.0001966740103, \quad z_{2} \approx 1.012034341
$$


Substituting values (10.101) into formulae (10.97) and (10.98), we arrive at the first approximation of the solution in question:

(10.102)

(10.103)

$$
\begin{aligned}
X_{11}(t) \approx & -0.0001966740103+0.012614706 t \\
& -0.00000001657742843 t^{7}-0.0002388490229 t^{5} \\
& -1.024213507 t^{3} \\
X_{12}(t) \approx & 1.012034341+0.02357590346 t \\
& -0.01234567901 t^{9}+0.06944444444 t^{8} \\
& -0.01418192059 t^{7}-0.6579145336 t^{6} \\
& +1.911974201 t^{5}-2.500341335 t^{4} \\
& +1.684429372 t^{3}-0.5044437784 t^{2}, \quad t \in[0,1] .
\end{aligned}
$$

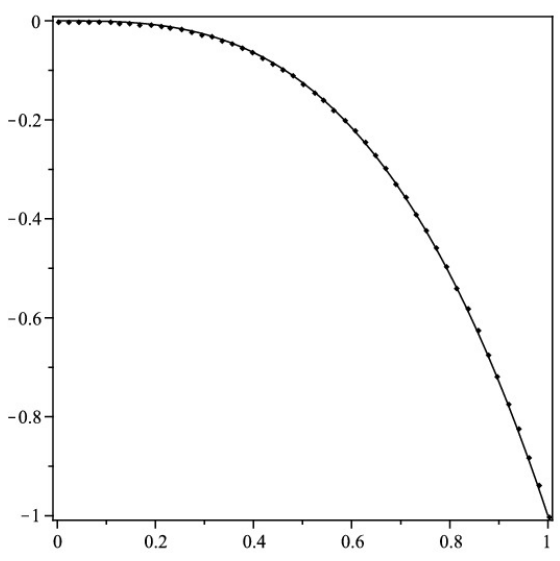

(a) First component

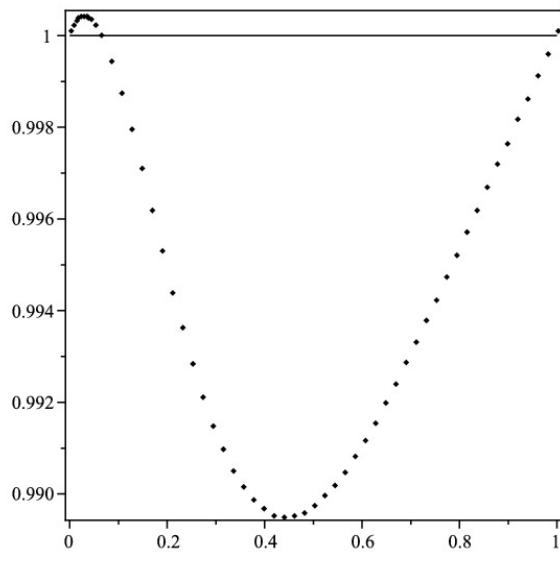

(b) Second component

FIGURE 1. The first approximation $(\downarrow)$ and the exact solution (solid line)

Note that, as can easily be verified, the pair of functions

$$
x_{1}(t)=-t^{3}, \quad x_{2}(t)=1
$$

is a solution of the original problem. We can compare the graphs of functions (10.102), (10.103) and (10.104) on Figure 1 .

Proceeding similarly, we find the roots of the second approximate determining equation

$$
z_{1} \approx 0.0001948022964, \quad z_{2} \approx 1.000364679
$$


which determine the second approximation of the solution:

$$
\begin{aligned}
X_{21}(t) \approx & -0.2362794075 \cdot 10^{-5}-0.121067348 \cdot 10^{-1} t \\
& -0.1172429156 \cdot 10^{-4} t^{39}+0.1390279168 \cdot 10^{-3} t^{37} \\
& -0.4469102669 \cdot 10^{-3} t^{35}-0.1254983549 \cdot 10^{-2} t^{33} \\
& +0.1320307360 \cdot 10^{-1} t^{31}-0.3558503833 \cdot 10^{-1} t^{29} \\
& +0.2690720263 \cdot 10^{-2} t^{27}+0.2552538119 t^{25}-.08676295819 t^{23} \\
& +1.644128081 t^{21}-2.083909660 t^{19}+1.799046652 t^{17} \\
& -0.8087453689 t^{15}-0.4604246775 t^{13}+1.259222257 t^{11} \\
& -1.103551748 t^{9}+0.4284412737 t^{7}-0.2831082023 \cdot 10^{-1} t^{5} \\
& -1.000290553 t^{3}, \\
X_{22}(t) \approx & 1.000145266-0.7063123523 \cdot 10^{-5} t+2.524666867 \cdot 10^{-16} t^{13} \\
& -1.367527886 \cdot 10^{-15} t^{12}+3.182679494 \cdot 10^{-9} t^{11} \\
& -1.750472409 \cdot 10^{-8} t^{10}+0.3625977159 \cdot 10^{-5} t^{9} \\
& -0.2022107430 \cdot 10^{-4} t^{8}+0.2464657817 \cdot 10^{-4} t^{7} \\
& +0.7277439546 \cdot 10^{-4} t^{6}-0.2757766822 \cdot 10^{-3} t^{5} \\
& +0.3858650306 \cdot 10^{-3} t^{4}-0.2744379408 \cdot 10^{-3} t^{3} \\
& +0.9296395556 \cdot 10^{-4} t^{2}
\end{aligned}
$$

(10.106)

for $t \in[0,1]$.

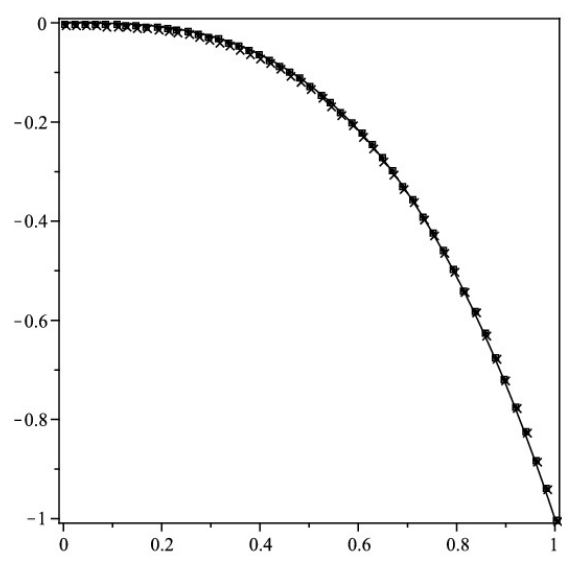

(a) First component

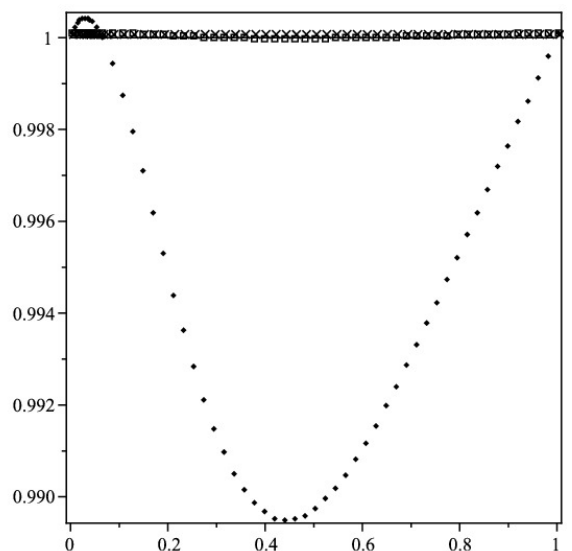

(b) Second component

FIGURE 2. The first $(\downarrow)$, second $(\times)$, and third $(\square)$ approximations of the exact solution (drawn with a solid line)

In a similar way, we find the third approximation, which has the initial data

$$
z_{1} \approx-0.000002362794075, \quad z_{2} \approx 1.000145266 \text {. }
$$


The graphs of the exact solution (10.104) and its first three approximations are shown on Figure 2 We see that the scheme applied here gives approximations which provide quite a good accuracy. In particular, the error of the third approximation does not exceed $10^{-4}$.

Acknowledgements. Supported in part by the Academy of Sciences of the Czech Republic through the Institutional Research Plan No. AV0Z10190503 (A. Rontó). This research was carried out as part of the TAMOP-4.2.1.B-10/2/KONV-2010-0001 project with support by the European Union, co-financed by the European Social Fund.

\section{REFERENCES}

[1] Berinde, V. Iterative Approximation of Fixed Points, second ed., Lecture Notes in Mathematics, 1912, SpringerVerlag, Berlin, 2007

[2] Krasnoselskii, M. A. and Zabreiko, P. P., Geometrical Methods of Nonlinear Analysis, Springer-Verlag, Berlin, 1984

[3] Mawhin, J., Topological Degree Methods in Nonlinear Boundary Value Problems, CBMS Regional Conference Series in Mathematics, 40, Amer. Math. Soc., Providence, R.I., 1979, Expository lectures from the CBMS Regional Conference held at Harvey Mudd College, Claremont, Calif., June 9-15, 1977

[4] Rontó, A. and Rontó, M., Successive Approximation Techniques in Non-Linear Boundary Value Problems for Ordinary Differential Equations, Handbook of Differential Equations, Ordinary Differential Equations, IV, Elsevier /North-Holland, Amsterdam, 2008, 441-592

[5] Rontó, A. and Rontó, M., On a Cauchy-Nicoletti type three-point boundary value problem for linear differential equations with argument deviations, Miskolc Math. Notes, 10 (2009), No. 2, 173-205

[6] Rontó, A. and Rontó, M., Successive approximation method for some linear boundary value problems for differential equations with a special type of argument deviation, Miskolc Math. Notes, 10 (2009), No. 1, 69-95

[7] Rontó, A. and Rontó, M., On nonseparated three-point boundary value problems for linear functional differential equations, Abstr. Appl. Anal., (2011), Art. ID 326052, 22 pages

[8] Rontó, A. and Rontó, M., Existence results for three-point boundary value problems for systems of linear functional differential equations, Carpathian J. Math., 28 (2012), No. 1, 163-182

[9] Rontó, A., Rontó, M., and Shchobak, N., On finding solutions of two-point boundary value problems for a class of non-linear functional differential systems, Electronic J. Qualit. Theory Different. Equat., (2011), No. 13, 1-17

[10] Rontó, M. and Samoilenko, A. M., Numerical-Analytic Methods in the Theory of Boundary-Value Problems, World Scientific Publishing Co. Inc., River Edge, NJ, 2000

[11] Rus, I. A., Some nonlinear functional differential and integral equations, via weakly Picard operator theory: a survey, Carpathian J. Math., 26 (2010), No. 2, 230-258

\section{INSTITUTE OF MATHEMATICS}

ACADEMY OF SCIENCES OF CZECH REPUBLIC

ŽIŽKOVA 22, CZ-616 62, BRNO, CZECH REPUBLIC

E-mail address: ronto@math.cas.cz

DEPARTMENT OF ANALYSIS

UNIVERSITY OF MISKOLC

3515, MisKOLC-EGYETEMVÁROS

E-mail address: matronto@gold.uni-miskolc.hu 